painted hands present in the first and third zones are absent in the second zone, which could not, therefore, have been derived from the first zone-but it is a little difficult to follow this. He thinks that the engravings were made by Bushmen akin to those of the second zone.

This is a valuable book recording the work and views of many people. I should like to have found a mention of that wonderful corpus of paintings in the Transkei copied by Brother Otto of Marienhill Monastery. I wonder, too, if it is possible to isolate the art of the Republic from that of Southern Rhodesia - in fact we want a bigger work still! The colour photographs are excellent.

\section{C. Burkitrs}

\section{SOLAR FLARE PHENOMENA}

\section{Solar Flares}

By Henry J. Smith and Elske van Panhuys Smith. Pp. xii +322 . (New York: The Macmillan Company; London: Collier-Macmillan, 1963.) 100s.

$\mathrm{T}^{\mathrm{T}}$ is now more than 100 years since solar flares were 1 first observed, although, indeed, the intensely bright white patches of light close to sunspots which were seen by Carrington and Hodgson in 1859 were only recognized as such in retrospect. After this event, a great magnetic storm occurred on Earth and brilliant displays of the auroræ were seen in both hemispheres. These events on the Sun and the accompanying geophysical phenomena have been investigated extensively during tho past century, but our knowledge of them is essentially observa. tional. and not one of the many theories of the cause of flares or of the accompanying geophysical effects is yet regarded as satisfactory.

The availability of improved instruments for the investigation of the Sun, the vast development of solar radio astronomy, and the stimulus given to investigators by the International Geophysical Year have now produced a mass of detail which it is hard to assemble in an orderly manner. A book devoted to the topic of the solar flare phenomena is thereforo to be particularly wolcomed, especially whon it is so authoritative and comprehensive as Solur Flares by Henry and Elske Smith of Sacramento Peak.

The book begins with a well-written account of the nature of the solar atmosphere and of the various active phenomena which it displays. The second chapter, which occupies more than a third of the book, first describes the methods and instruments used for examining solar flares and then becomes a source book of information about the flare data; classification, relation to spots, location on the disk, distributions and asymmetries, durations, structures, light and growth curves and many similar data are included.

The third chapter, which gives a similar treatment for the spectral characteristics of the flares, concludes the part of the book devoted to the optical observations of the flares themselves. However, for many readers, the last three chapters may well prove to bo the most interesting in the volume. The authors are careful to emphasize that they make no claim other than to have summarizod the available data on the radio emission and on the solar corpuscular emission and geophysical phenomena in two of these chapters, but this they have done exceedingly thoroughly. Fven radio astronomers will find the chapter on radio emission a most convenient and expert summary of a part of their subject which has recently grown so rapidly that comprehension is difficult.

The geophysical chapter covers a wide field and includes the cosmic-ray effects, space radiation hazards, geomagnetic field variations and the aurora and ionospheric phenomena associated with solar flares. Finally, the authors deal with the nature of flares and discuss the various possible theories of their origin. To me the last few pages in the book wore of outstanding interest. Here the authors discuss the possible nature of similar activity on the stars. In view of the recent work on the radio emission from flare stars this may woll be the only section of this excellent book which will need revision in the noar. future. BERNARD J.OVELL

\section{GAS-COOLED REACTOR TECHNOLOGY}

The Design of Gas-Cooled Graphite-Moderated Reactors Edited by D. R. Poulter. Pp. xii $+692+13$ plates. (London: Oxford University Press, 1963.) 84s. net.

G have been operating the United Kingdom they have a than a decade and in of millions of pounds and have had committed to them a significant proportion of the Nation's electricity supply capacity. Against this background a comprehensive textbook on the subject was long overdue. The present volume, written by a number of specialists mainly from the Reactor Group of the United Kingdom Atomic Energy Authority, sets out to cover the field, particularly, according to the preface, for young postgraduate engineers or those just entering nuclear engineering. It is therefore a primer, not a design manual. The sixteen chapters encompass basic concepts such as reactor physics and materials technology, specific topics, such as fuel handling, instrumentation and civil and structural engineering, and end with an outline of possible future developments. There are five appendixes containing nomenclature and some of the more mathematical parts of the text.

To achieve uniform presentation is a well-known difficulty of joint authorship. It requires, at least, a common assumption about the basic knowledge and the intellectual level of the intended readers and sufficient co-ordination to avoid repetition: neither requirement appears to have been met satisfactorily in this volume. The opening chapter on the design concept, which could have been used to set the scene, is written rather curiously from an academic rather than an engineering point of view and does not connect the reader very well with what follows. It contains statements such as "other methods of power generation exist" which aro unlikely to surprise the student although he may easily gain the impression that otherwise no attempt would be made to design nuclear plant for minimum cost. Thus begun, the book proceeds with a fairly logical sequence of contributions, some of which are clear and authoritative. Steady-state reactor physies and reactor kinetics are well, if not simply, presented and there is a carefully compiled chapter on materials technology in which the sections on steels and graphite are worthy of mention and that concerning fueleloment materials, although at a disadvantage on account of its wide scope, provides a good technological primer. Only in the case of the steels, however, is the difference between fundamental and structure-sensitive properties discussed. In contrast, plant safety is given only a brief chapter, principally occupied with fault conditions, which ignores the important boaring that safety requirements have on plant design and economics and also oversimplifies health physics considerations. An allied subject, reactor shielding, is treated with scant regard for fundamentals and contains a number of errors not oasily put down to typographical mistakes. In this way one can proceed through the text weighing pros and cons in an effort to reach an overall assessment of the value of the book.

The book is ambitious in concept, but as a wholo must be judged disappointing and failing to reach its declared objective. Where a theme is discernible it seems to concern both the noed to keep down capital costs and the 